\title{
Design Feasibility Study of PET/CT Facility in
} Iraq

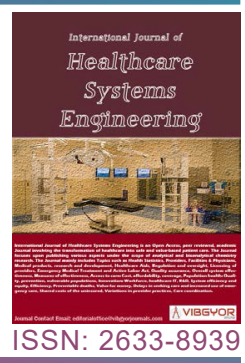

\section{Hassanain Ali Lafta* and Shatha Yaseen Zamil}

Biomedical Engineering Department, College of Engineering, Al-Nahrain University, Iraq

\begin{abstract}
Positron Emission Tomography-Computed Tomography (PET/CT) framework is a cutting-edge imaging tool in nuclear medicine. The essential objective of PET/CT imaging is to create profoundly exact combination images with a good enrolment of both CT and PET images on the same platform. Such major specialized equipment requires careful structural designs, and planning considerations of the functional components to facilitate the traffic of patients, staff, and radioactive materials. The main purpose of conducting this study is twofold; to give insights into the planning and design criteria of a typical PET/CT facility and to explore the impact of such criteria on the patient's attitude towards the experience and satisfaction with the examination at one of the most important facilities in Baghdad City, the Baghdad Center for Radiation Therapy and Nuclear Medicine. Some challenges were investigated in this center. Also, a cross-sectional survey was conducted on 88 patients who were scheduled for a PET/CT examination at the Baghdad center. All the participants supported the idea that the new PET/CT facility at Baghdad Medical City with considering that it must be planned and designed on the basis of regulatory requirements and radiation safety standards. The obtained findings indicate the paramount importance of such a qualitative study. This will significantly impact the management of patients and reduce both cost and potential associated with their traveling to the facilities that existed in the neighboring countries. Such findings might be utilized to help optimize such medical protocols and patient consideration as well. In the future, further research is required to identify which information demands are crucial for improving the cancer care facilities in Iraq.
\end{abstract}

\section{Keywords}

$\mathrm{PET} / \mathrm{CT}$, Functional areas, Shielding, Workflow, Patient satisfaction

\section{Introduction}

PET (Positron Emission Tomography) has been effectively used to detect cancer. With the accuracy of the imaging result, this technology is becoming the most useful forms of diagnostic imaging. Instead of the quality of PET scan for imaging, the effectiveness of PET scan can be improved if it combined with CT (Computed
Tomography) scan to perform a PET/CT scan. From that point, the progress of this technology has been spread over throughout the world. The use of the cyclotron to obtain the radioactive substance (18Ffluorodeoxyglucose) for PET scan has been improved linearly with the progress of PET scan improvement. The main problem of PET and CT combinations into a PET/CT scan is that this device is costly. However, since the accuracy of PET/CT

*Corresponding author: Hassanain Ali Lafta, Biomedical Engineering Department, College of Engineering, Al-Nahrain University, Baghdad, Iraq

Accepted: December 29, 2021; Published: December 30, 2021

Copyright: (c) 2022 Lafta HA, et al. This is an open-access article distributed under the terms of the Creative Commons Attribution License, which permits unrestricted use, distribution, and reproduction in any medium, provided the original author and source are credited.

Lafta et al. Int J Healthc Syst Eng 2022, 3:009

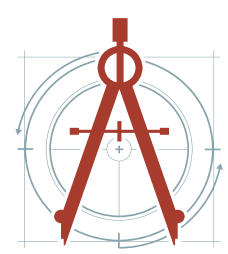


scan is undoubtedly much better than PET and CT scans separately, this technology preserves a great contribution of medical sciences [1].

Despite the fact that the framework can be operated to acquire either CT images or PET images, they are predominantly operated to acquire both, joining two clinical imaging advances: CT for anatomical imaging and PET for functional imaging. This brings the advantages and furthermore the complexities of the two frameworks while giving anatomical and functional information through fusion images. This technique involve two sources of radiation, the first one is gamma ray from the patient's body after being injected with the isotopes (radiopharmaceutical materials) and the second is the X-ray from the CT gantry. So the planning and the designing process must be done carefully [2].

For the planning and designing of a typical PET/ CT facility several standards must be followed in order to reduce the radiation scattering and guarantee more safety for the patients and staff as the radiations (gamma and X-Ray) are very dangerous. Shielding is important factor to provide safety against radiation scattering. PET/CT facilities required additional shielding as powerful radiations are emitted from two sources [2].

The planning deals with the layout of the facility, location as it affects the traffic of the patient, staff and the isotopes, and site planning that must be carefully planned in order to separate the cold area from the hot area. The design of the facility is a critical process that needs many of engineering specialties.

There are many things should take in consider when design the facility like the size of rooms, weight of the equipments and their distribution and shielding requirements. As gamma ray has potential hazards on the human body because its short wavelength and powerful body penetration, shielding must be effectively done in order to reduce the scattering of these rays [2].

Moreover, patient satisfaction has received great attention today and has become one of the main concerns of any healthcare facilities. Any organizations and building operations need the users' opinion to improve its efficiency. Because patients are the main priority of any healthcare facility, they are considered as a reliable resource in providing feasible information to understand quality. Efforts should be made to make the patients' stay as comfortable as possible in order to improve their satisfaction.

The main purpose of conducting this study is twofold; to give insights into the planning and design criteria need to be addressed for a typical $\mathrm{PET} / \mathrm{CT}$ facility, as well as to explore how such criteria will influence the patient's satisfaction and attitude towards the examination experience. This was carried out at one of the most important facilities existed locally in Baghdad Medical City which is Baghdad Center for Radiation Therapy and Nuclear Medicine, through reviewing the unit layout design, functional areas and organization of required equipment and relationships between these areas inside the facility in accordance with the regulatory requirements and radiation safety standards. Authorization and permission were obtained from the dedicated institution before conducting this study. Qualitative review was performed under the supervision of the technical and administrative concerned inside the facility.

Building a new PET/CT facility de novo or setting one up in an existing nuclear medicine or radiology department requires a good planning team [3]. Such facilities are frequently under duress in order to meet the demands of extreme situations. As a result, this facility should be carefully designed and constructed to guarantee that the spaces are comfortable for users, particularly patients. Basically, a proper design should be able to improve the organization's clinical, economic, productivity, satisfaction, and cultural measures. Quality design indicators can be measured fairly based on subjective opinions, experiences and preferences.

International Atomic Energy Agency (IAEA) and other worldwide associations have created quality standards and guidelines that endorsed the maximum radiation exposure limit for the experts, the overall population, and the environment [4]. Appropriate layout and work process plan of nuclear medicine facility, alongside ideal isotope obtainment and utilization arranging may diminish the radiation trouble on the experts, the patients, the overall population, and the environment everywhere [5].

The shielding of PET/CT facilities present special challenges because of the $511 \mathrm{keV}$ annihilation photons associated with the positron decay. These emissions are much higher in energy than those 
typical radionuclide used in other diagnostic nuclear medicine procedures. As a result, considerable barrier shielding is required in floors, ceilings and adjacent walls. Once the radiopharmaceutical is administered, the patient becomes the radioactive source and continues to be one for the duration of his stay in the facility. Performing shielding calculations at a near boundary requires knowledge of the distance from the source of radiation to the nearest occupants; the maximum expected workload; the levels of occupancy in nearby areas and the type of radiation involved and particular approaches used in nuclear medicine [6].

\section{Baghdad Center for Radiation Therapy and Nuclear Medicine}

This center is one of the most important PET/CT facilities in Iraq as it is included within the largest health care facility in the county, Baghdad Medical City, where composed of fifteen specialized hospitals and medical centers. This centre was established in 2015 and composed of two floors; and evolved medical imaging modalities used for diagnostic and therapeutic purposes for cancer care. The PET/CT equipment was installed in 2018 and starts working in 2019. The centre receives about 20-30 patients monthly and it does not support the patient stay for twenty-four hours, see Figure 1.

\section{Functional relationships}

It is crucial for the success of the facility to organise a floor plan taking into account how the rooms and spaces will be distributed according to the preformed functions per each area, the flows and radiation protection measures. According to the risk and level of radiation exposure, in the following different functions will be allocated to areas with either a low risk of significant radiation exposure (so-called cold or uncontrolled areas) or with high risk of radiation exposure (so-called hot or controlled areas) [2].

Typically, PET/CT area may be located on the ground level with easy access from the main entry area for patients, staff and visitors. Externally, this area should have good access to the entry point of the Hot Lab room for delivery of externally provided radioisotopes in a route as direct as possible. Also, there should be a good access to the support and service units such as clinical information, housekeeping and supply via a service corridor with entry to the un-dosed patients' area. The support units should be located centrally to the scanning and patient areas for maximum convenience [7].

An all around planned facility incorporates a separate hot laboratory for radiopharmaceutical storage, adjustment, and dispensing, and properly

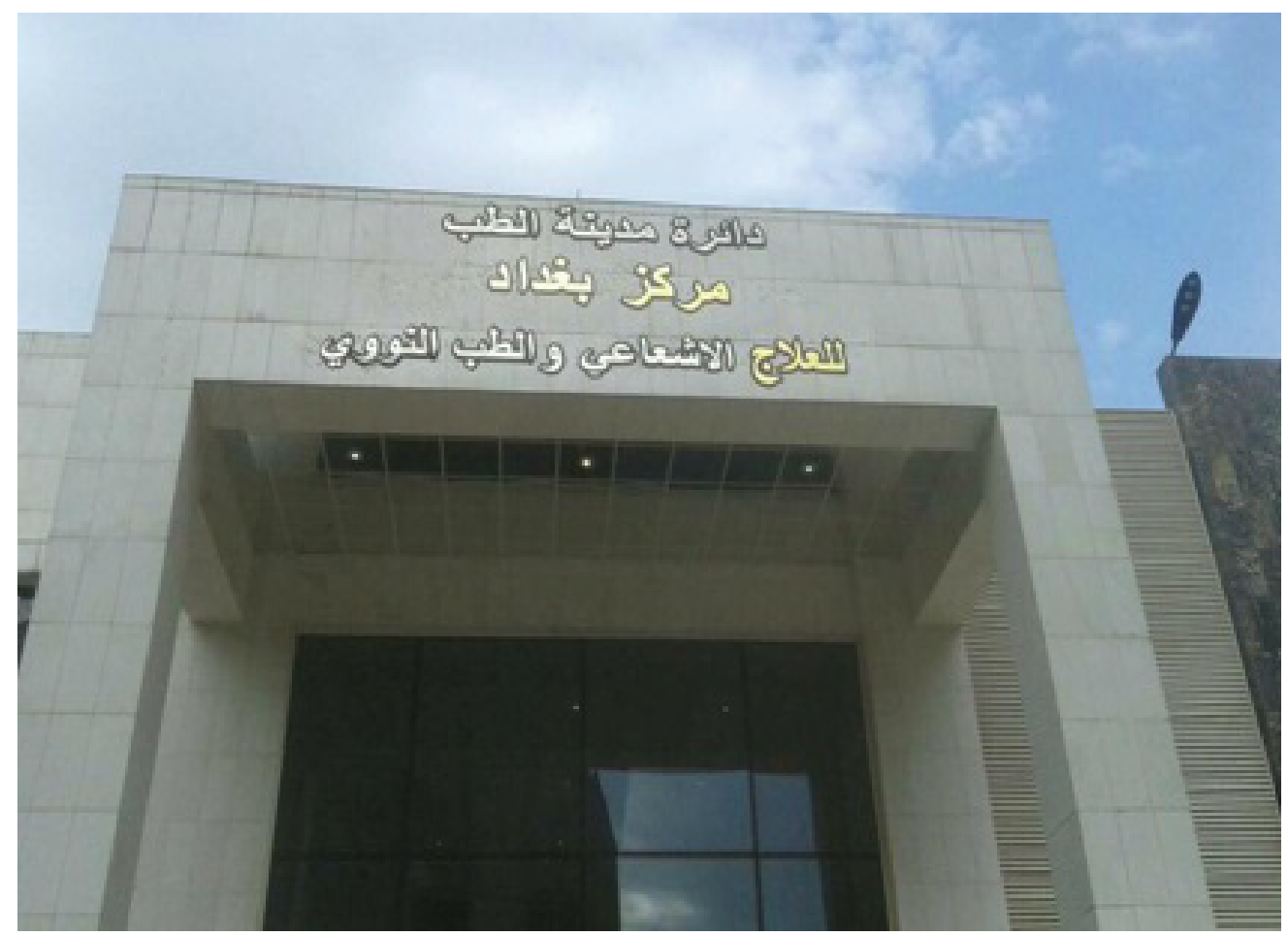

Figure 1: The front entry of Baghdad center for radiation therapy and nuclear medicine. 


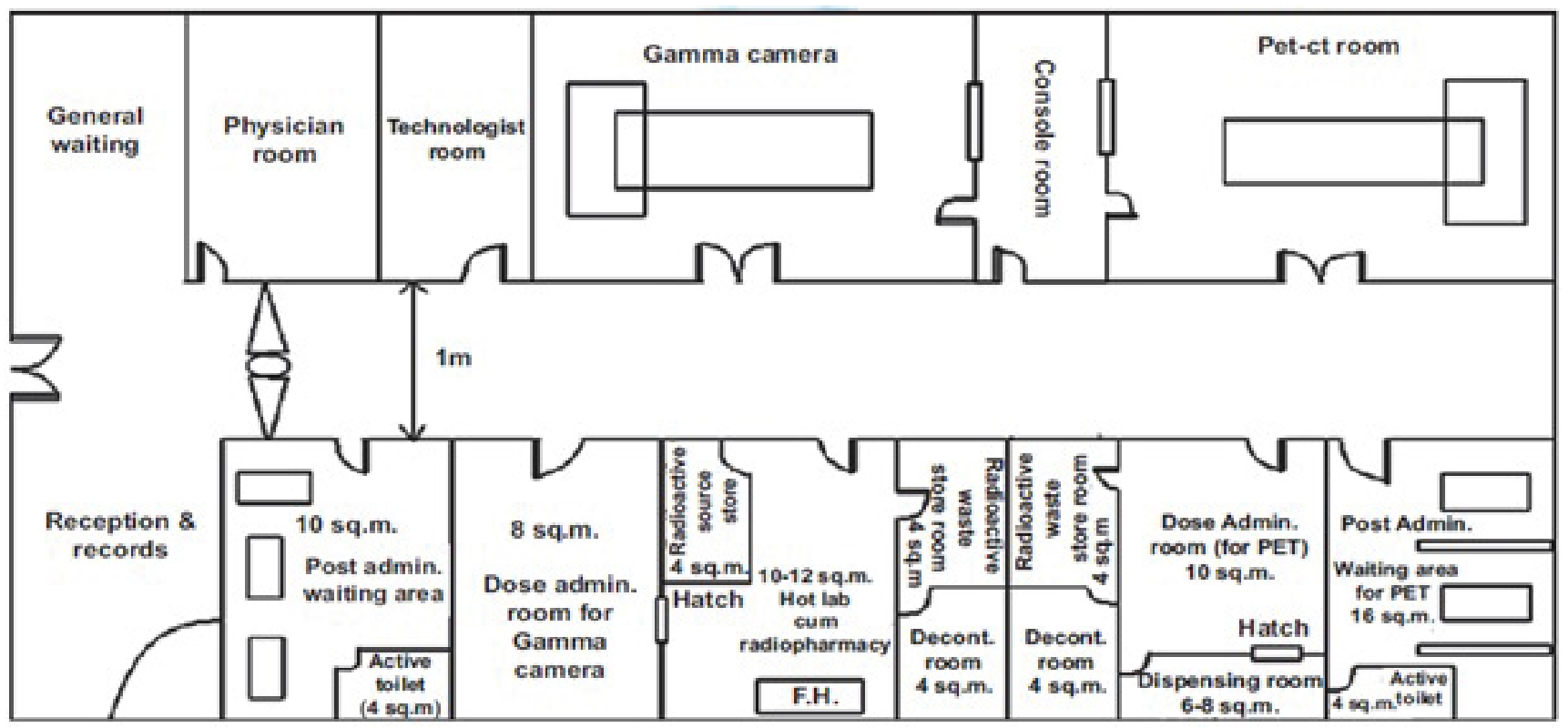

Figure 2: A typical layout for nuclear medicine facility having both gamma camera and positron emission tomography-computed tomography (PET/CT) installations [8].

protected waste storage area/container(s), and separate patient injection and holding spaces. Careful consideration ought to be paid to the zone of the administering and patient injection areas, the post-injection holding rooms, and the patient toilet according to zones utilized by the overall population and staff. The distances from injected patients and additionally sources to protected walls ought to be expanded to restrict the amount and cost of required protecting. Figure 2 shows a potential plan that exploits the area layout to separate staff and locators from the high exposure patient uptake zone. The patient toilet is effectively accessible and near where it will be required by and by [8].

The PET/CT center should be divided into three adjacent sections; one for the scanner; one for the cyclotron; and the third for the office area. The size of the first two sections depends on the dimensions of each vendor's equipment, and the third section depends on the size and scope of the operation in a given. Internally, PET/CT area may generally be a devoted set-up of rooms inside a larger unit typically specialized in nuclear medicine yet in addition might be a part of a coordinated clinical imaging unit. The reception will give an entrance control point and there will be away from of undosed and dosed patient areas of the facility. There ought to be a make way of movement for patients who show up and stand by in un-dosed waiting, at that point are moved to uptake rooms dosing, wait for take-up followed by scanning procedures, then return to uptake rooms for a cool-down period waiting for radioactivity to dissipate prior to discharge, preferably through a different exit, and not through zones where un-dosed patients and visitors are waiting. The ideal relationships should maintain the reception at the entrance to provide access control, with direct view of waiting areas. Also, there should be separation and access control between un-dosed areas and dosed areas of the unit [7].

\section{Functional areas}

The PET/CT modality was installed in the second floor of the center building. This floor was designed to involve the following functional areas:

Reception room: The reception room is specified for the reception of the patients who are referred or wanted to book for scanning appointments, inquiring about the scanning procedure and what to do before and after it, see Figure 3 . As patients arrive, they are received and logged in by the administrative staff. Handouts and pamphlets with general information about the PET/CT exam and particular recommendations that apply to their specific output ought to be given and accessible to peruse while waiting. Typically, the reception room is located at the front of the facility, normally with the secretarial room to its rear. The two zones need 


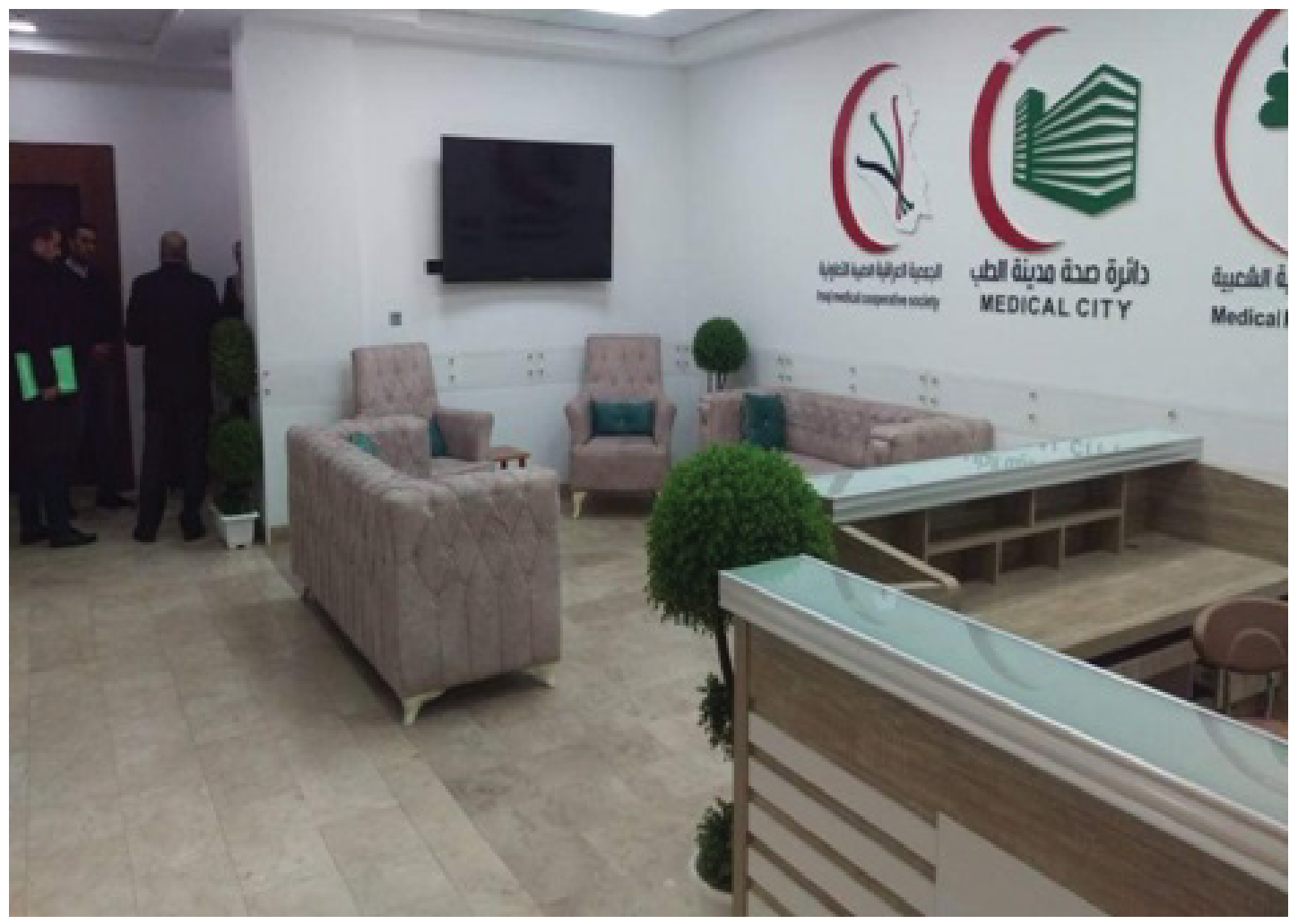

Figure 3: The reception room in Baghdad center for radiation therapy and nuclear medicine.

between 10-20 square meters, depending on the workload.

Consulting room: In this room, the request and clinical records are analysed and the patient is interviewed and physically examined, if necessary. The patient is educated about the idea of the particular assessment he/she is going through. This room ought to be near the waiting room and satisfactorily prepared. A supply of oxygen gas for clinical use and vacuum for aspiration and all different administrations according to the local regulations ought to be provided. An area of not less than 12 square meters is fundamental. A specific number of rooms ought to be accessible for clinical, scientific, and technical staff, and for meetings and teaching activities, the number depending on the size and aims of the unit [6].

Control room: This room is used to control operation of the device and monitor the patient condition during the scanning procedure. This can be administrated by a physiotherapist. The walls of this room were shielded with concrete and gypsum, while the floor was shielded with $35 \mathrm{~cm}$ of concrete in addition to $6 \mathrm{~mm}$ of lead. The door was shielded with $6 \mathrm{~mm}$ of lead. The room employed two computers for the operator and the physician with $C D$ printer and paper printer. Close monitoring of staff exposures was maintained. It utilized the console and radiation dosimeter devices mounted on the room walls in order to detect the radiation percentage that the staff exposed to. The room also contains two emergency bottoms, one to stop the device motors and the other to stop the device completely depending on the existing emergency degree, see Figure 4.

Control and scan rooms: This room is composed of the PET/CT components which involved; the patient gantry, the stabilizers which are used for stabilizing the paediatric patients, the main power supply, the power distribution unit (PDU) which represents a large power strip without surge protection. It contains monitor for monitoring the patient's physiological vital signals (by means the heart and lung functions) during the scanning. Moreover, there was a lead-glass panel between the control room and scan room for monitoring the patient. The room was also occupied with a camera for wider view for the scanning room, see Figure 5.

This room represents the facility heart. The scanning room ought to be effectively reached from the preparation rooms and the toilet. The door is typically before the preparation block. 

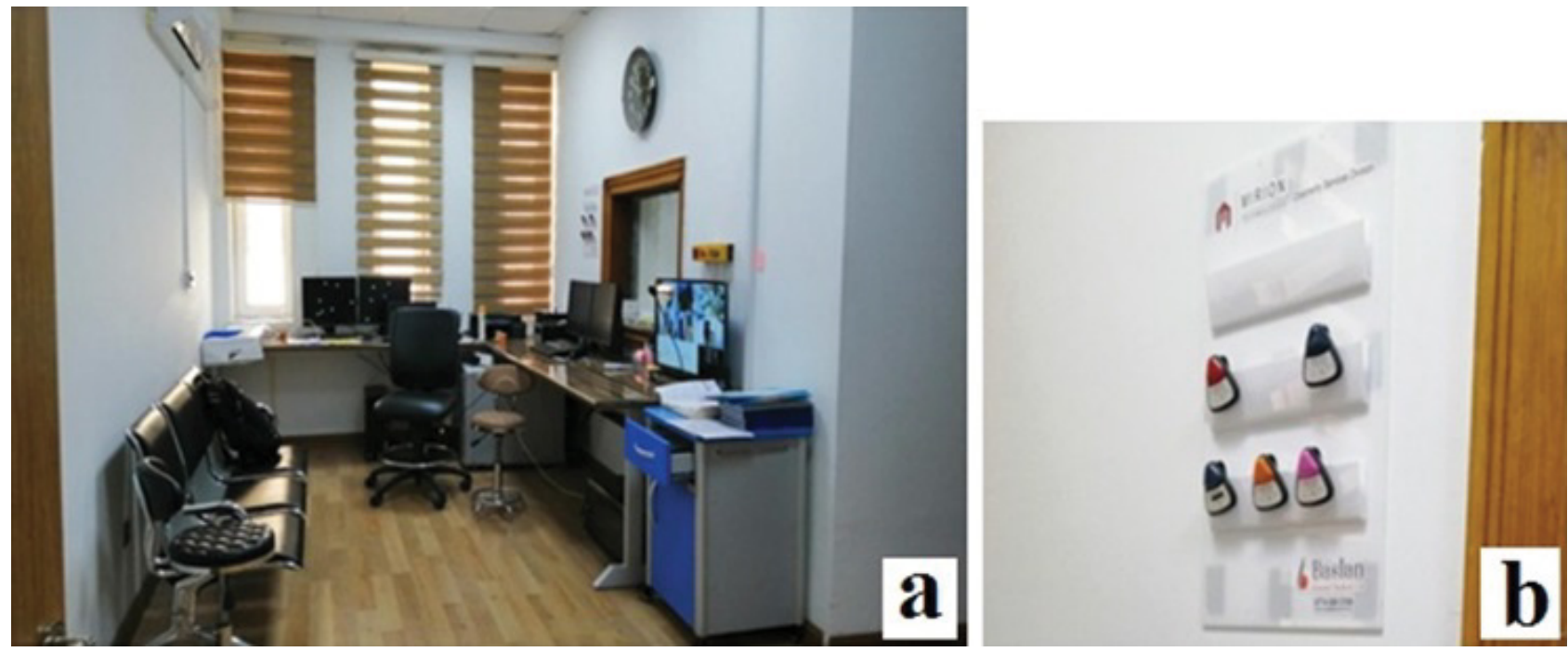

Figure 4: The control room in Baghdad center for radiation therapy and nuclear medicine: a) Control monitors; b) Radiation dosimeters.
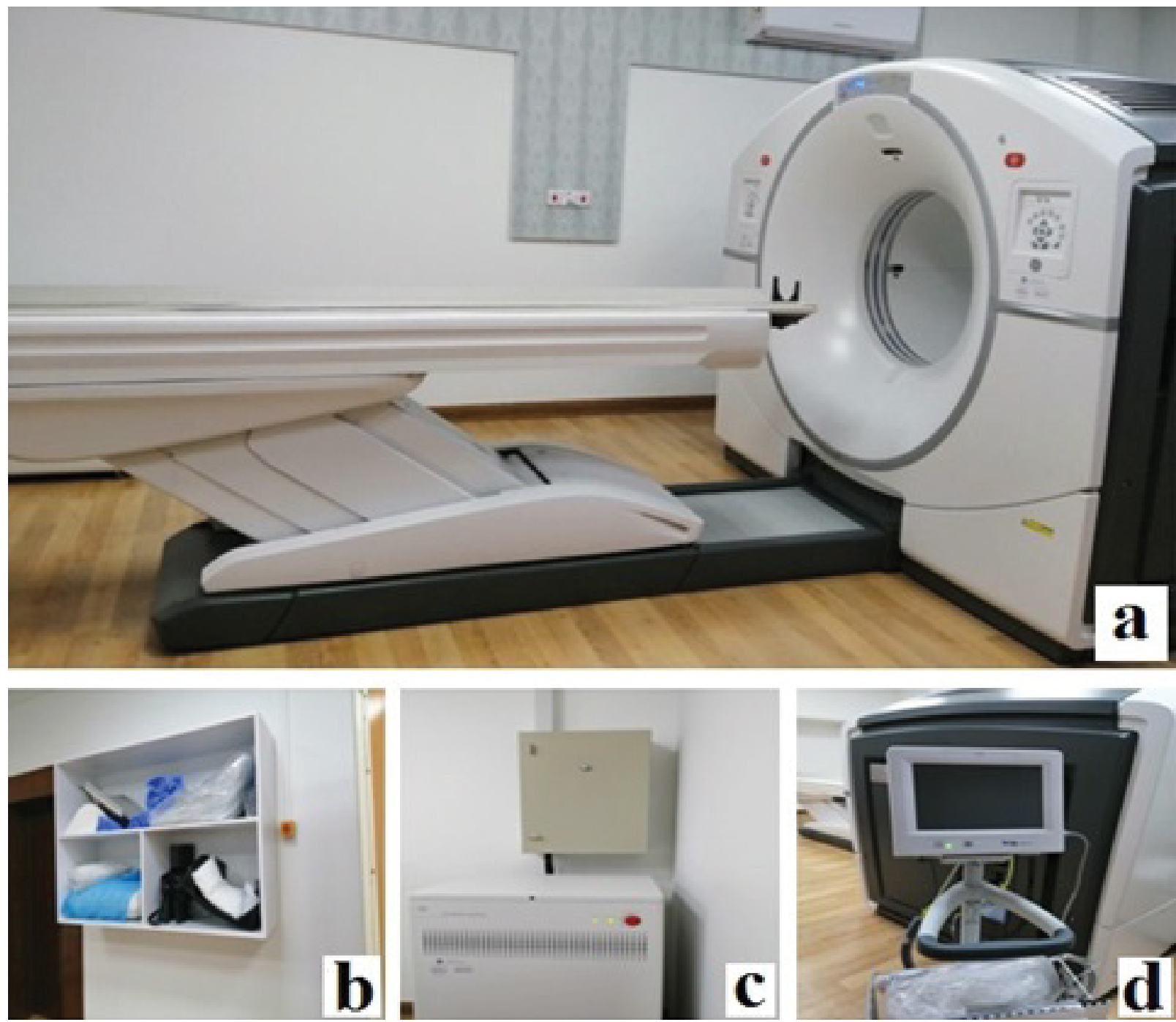

Figure 5: The scan room in Baghdad center for radiation therapy and nuclear medicine: a) PET/CT equipment; b) The stabilizers; c) The PDU and power supply; d) The monitor. 
In spite of the fact that the region required for proper installation of a PET/CT scanner can be as small as 35 square meters, some additional room will ease diagnostic as well as maintenance operations. Vendors' prerequisites and installation guidelines to be considered in the planning phase. Additionally, careful consideration ought to be given to the way that PET/CT scanners are to some degree requesting as far as site requirements: the gantry of a multi-modality scanner could weigh in excess of $3000 \mathrm{~kg}$. The walls of this room were shielded with concrete and gypsum, while the floor was shielded with $35 \mathrm{~cm}$ of concrete in addition to $6 \mathrm{~mm}$ of lead. The door was shielded with $6 \mathrm{~mm}$ of lead. It designed to provide standard electrical outlets for the data center equipment and has no monitoring or remote access capabilities.

Injection room: Injection/preparation rooms should be available to host three to four patients (not less than roughly 12-16 square meters) for each PET/CT scanner installed. Patients after injection are a relatively intense source of radiation (of the order of $30-50 \mu \mathrm{Sv} / \mathrm{h}$ per patient at 100 $\mathrm{cm}$ just after the administration). The assembly of several patients in the uptake room areas is a radiation protection problem that should not be overlooked; proper positioning and shielding of the uptake rooms need particular attention. There are two types of radiation sources present in the nuclear medicine department: (1) Radioisotope and (2) The injected patients. The radioisotope present in the department can be confined and shielded to achieve negligible radiation exposure from it when not in use [9]. This room is utilized before the scan to inject the patient with the radiopharmaceutical materials. It composed of two beds for the patient and the sanitation facilities, see Figure 6 . The walls of this room were shielded with concrete and gypsum, while the floor was shielded with $35 \mathrm{~cm}$ of concrete in addition to $6 \mathrm{~mm}$ of lead. The door was shielded with $6 \mathrm{~mm}$ of lead.

Patient waiting room: The patient waiting room is also equipped with patient bed and sanitation facilities, but it is utilized after the scanning where the patient still there until the radiopharmaceutical materials are totally exit from his body. This room is also shielded within the same way like the scan and the injection rooms, see Figure 7. The appointment timetable ought to take into consideration a waiting time of close to 30 minutes and if any deferral is likely patients ought to be informed. It ought to be considered that oncological outpatients as often

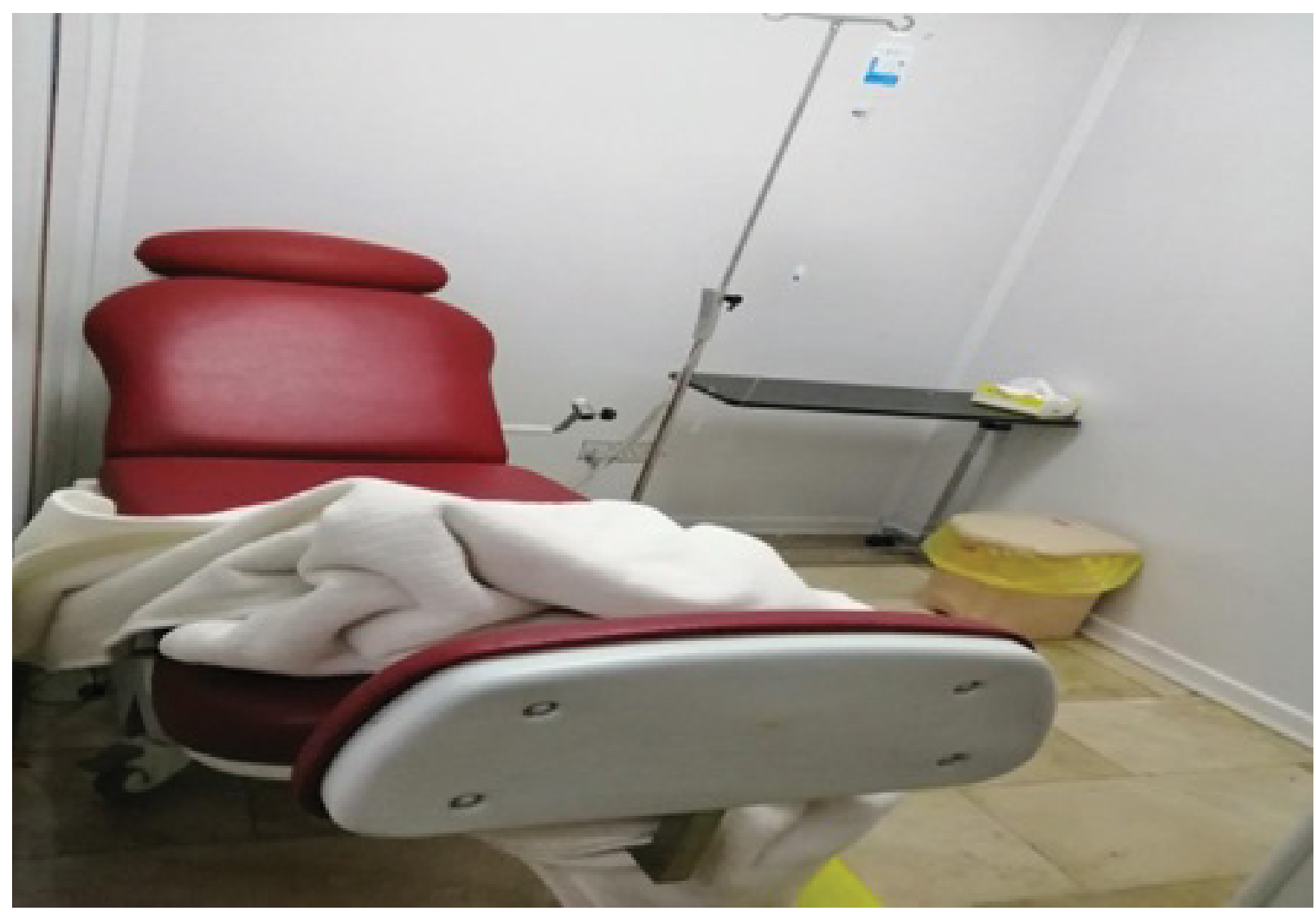

Figure 6: Patient bed inside the injection room in Baghdad center for radiation therapy and nuclear medicine. 


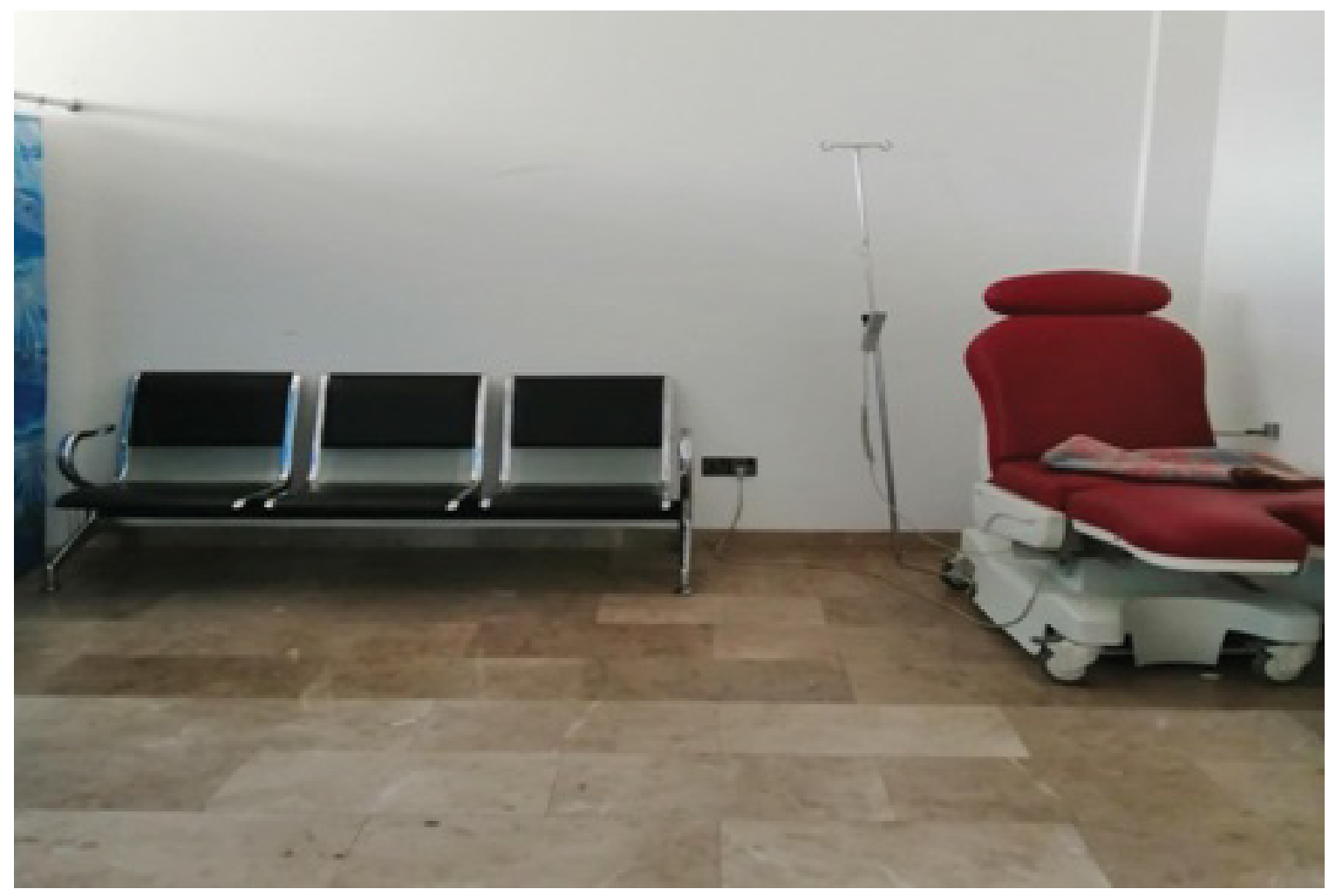

Figure 7: The patient waiting room in Baghdad center for radiation therapy and nuclear medicine.

as possible accompany a going with individual, thus the waiting room ought to be constructed accordingly. An area of no under 16 square meters is exhorted for a department with a single scanner. An area near the reception is recommended.

Hot lab room: The hot lab room is utilized for the examination of the radiopharmaceutical material activity and the estimation of its quantity that should be administered to the patient before the scan. This can be done by the dose calibrator instrument which is composed of an ionization chamber constructed to measure the number of ions within a medium. Regularly, PET radiopharmaceuticals can be administered to the injecting room in two different ways: either in a monodose syringe or in a vial. At the point when it is in a vial, the radioactivity might be high, contingent upon the number of patients, and each dose must be administered from the vial; for this situation, a small room, planned as a basic hot lab with protecting for positron producers and close to the injecting room, is required.

Reporting room: At the point when the procedure is accomplished, the examination is checked and the images analysed for reporting. There ought to be space for at any rate one preparing and fusion workstation, one for perception, a work area, and the commonplace furniture for diagnostic imaging. The space ought to be at the very least 10 square meters and it ought to be situated in similar zone as the workplaces.

Sanitation facilities: Both injection and patient waiting rooms should be equipped with sanitation facilities. When the patient is injected with the radiopharmaceutical material, he must wait for about 45-90 minutes which is the end of the uptake period. The patient is urged to urinate in order to reduce the amount of activity in the bladder. For that reason, there must be a sanitation facility in the injection room. Also, when the scan is finished, the patient must empty his body from the rest of the radiopharmaceutical materials through drinking lots of liquids would help the radioactivity to dissipate prior to discharge.

These facilities have lead reinforcing pipes that ended to reservoirs under the ground. Within the facility, adjacent toilet and preparation rooms are like an independent block that accomplishes specific functional and radiation protection requirements. About 30 square meters is sufficient for the entire block. The entire space needed for a facility holding fast to the above depiction is around 
170-200 square meters, of which about half will be 'controlled/restricted' zones, including the PET/CT block and the tracer administration block, while the other half will have activities which don't infer the utilization of any radioactivity, for example, workplaces and the reception block. Subsequently, should the facility be situated in an nuclear medicine department, about $40 \%$ of the space required could be considered as being for basic use, which would represent an extensive sparing in the financial plan allotted for development [2].

Cyclotron unit: In order to construct a cyclotron for the PET/CT scan equipment, there are constraints which need to be solved; financial, structural, political, conceptual, organisational, and human resources. The problem arises when one of those criteria is missing [7]. For Baghdad center, the cyclotron site is under construction and the radiopharmaceutical materials (isotopes) are not recently produced there. At the beginning, the provision of these materials was achieved from other resources outside the city, and then they were provided from other private hospitals in
Baghdad. The FDG (18F-fluorodeoxyglucose) had used as the essential radiopharmaceutical material for the scanning procedure in this center.

\section{Workflow Management}

Admittance to the facility for both ambulatory and gurney patients will be required. A few zones will be assigned as controlled areas, with access confined to PET/CT staff. Different areas might be assigned as supervised with access constrained by signage and notice lights. Access to staff only areas should be conceivable without going through high activity zones. Patients ought to have the option to enter and leave the facility without going through staff only regions. The layout ought to be with the end goal that it facilitates patient movement through the various steps involved. The exit route for patients post-scanning ought to be arranged so that, where conceivable, they leave the facility instantly without going through different offices or occupied public regions. On the off chance that the PET/CT scanner is to be situated in or near nuclear medicine, care must be taken to evade

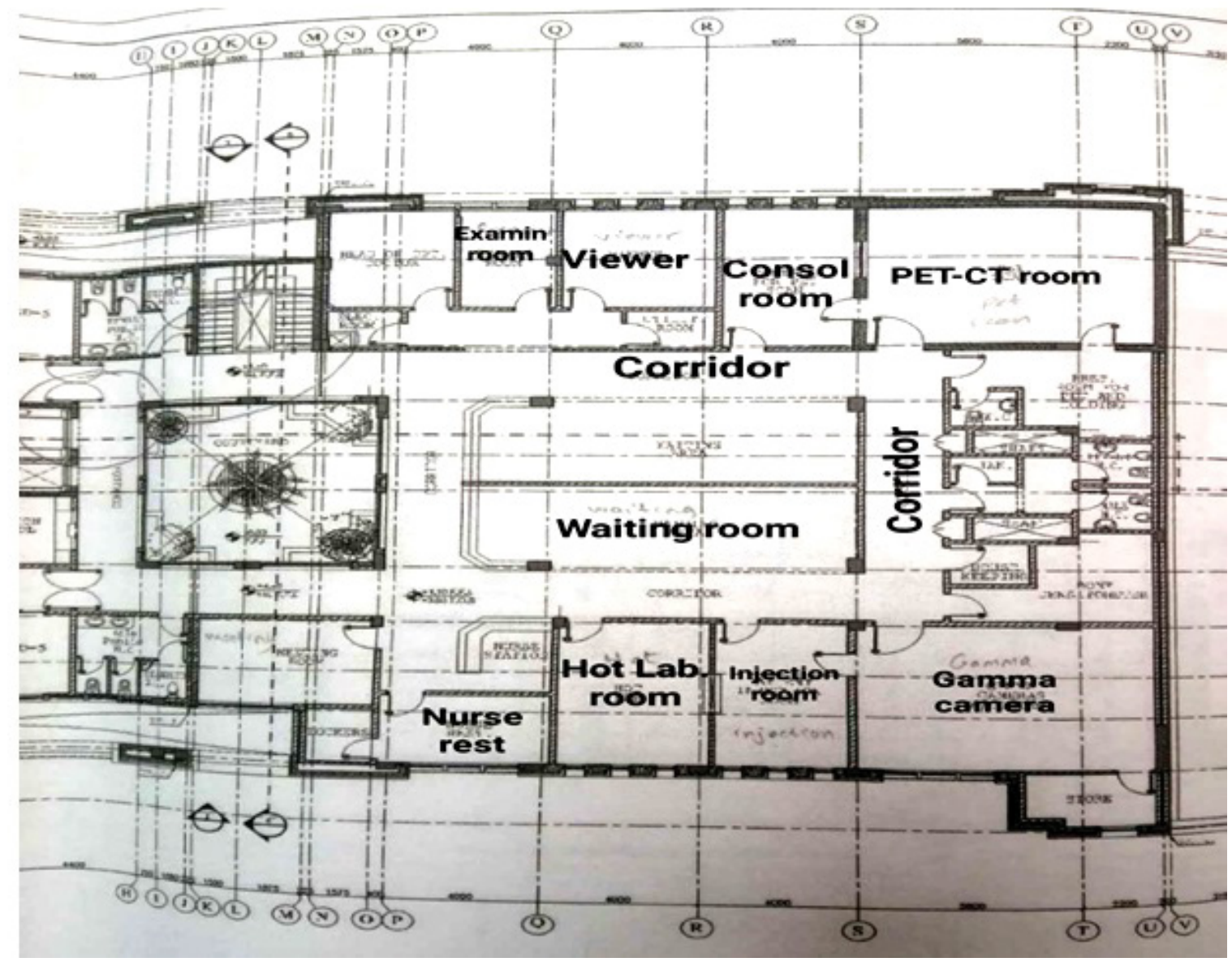

Figure 8: The site plan of Baghdad center for radiation therapy and nuclear medicine. 
obstruction from injected patients with other imaging equipment. Similarly, care must be taken that obstruction with delicate equipment, including gamma cameras, doesn't emerge as patients leave. Figure 8 shows the site plan of Baghdad center where describes the functional relationships of the components of the facility.

Planning and smoothing out the workflow appropriately and reliably can feasibly limit radiation exposure. Simultaneously, powerful sharing of the workload among the professionals may likewise be acquainted to limit individual radiation exposure. The patients referred for a PET/CT study are gone to at a devoted counter for appointments. Appointment is scheduled by the trained staff present at the counter and definite scan-specific directions are given to the patient at the hour of arrangement. On the appointed date, the patient reports at nursing station where the nurse in charge confirms the patient details and tests endorsed, and refers him/her to the resident doctors for recording significant clinical history. Relevant instructions are given to the patient with respect to the prescan and postscan precautions.
$\mathrm{He} / \mathrm{she}$ at that point continues to the injection room, see the Figure 9.

\section{Materials and Method}

The most important measure in any evaluation of any building's design feasibility is whether it satisfies user requirements and what users think and feel about it. Patient's satisfaction provides a significant result measure of quality care for improving consistence to treatment regimens. A cross-sectional survey was conducted to a patient sample of 88 participants, $(52$ males and 36 females), with mean age (48.69 \pm 7.31$)$ years, who were scheduled for a PET/CT examination at Baghdad center and asked to participate in this survey after completion of their examination in order to explore their attitude about experience of the assessment. The survey comprised of inquiries concerning individual patients' information like age, gender and profession besides their level of satisfaction. The participants enrolled in this study were subsequent to giving their informed consents in accordance with the ethical approval obtained from Al-Nahrain university ethics committee.

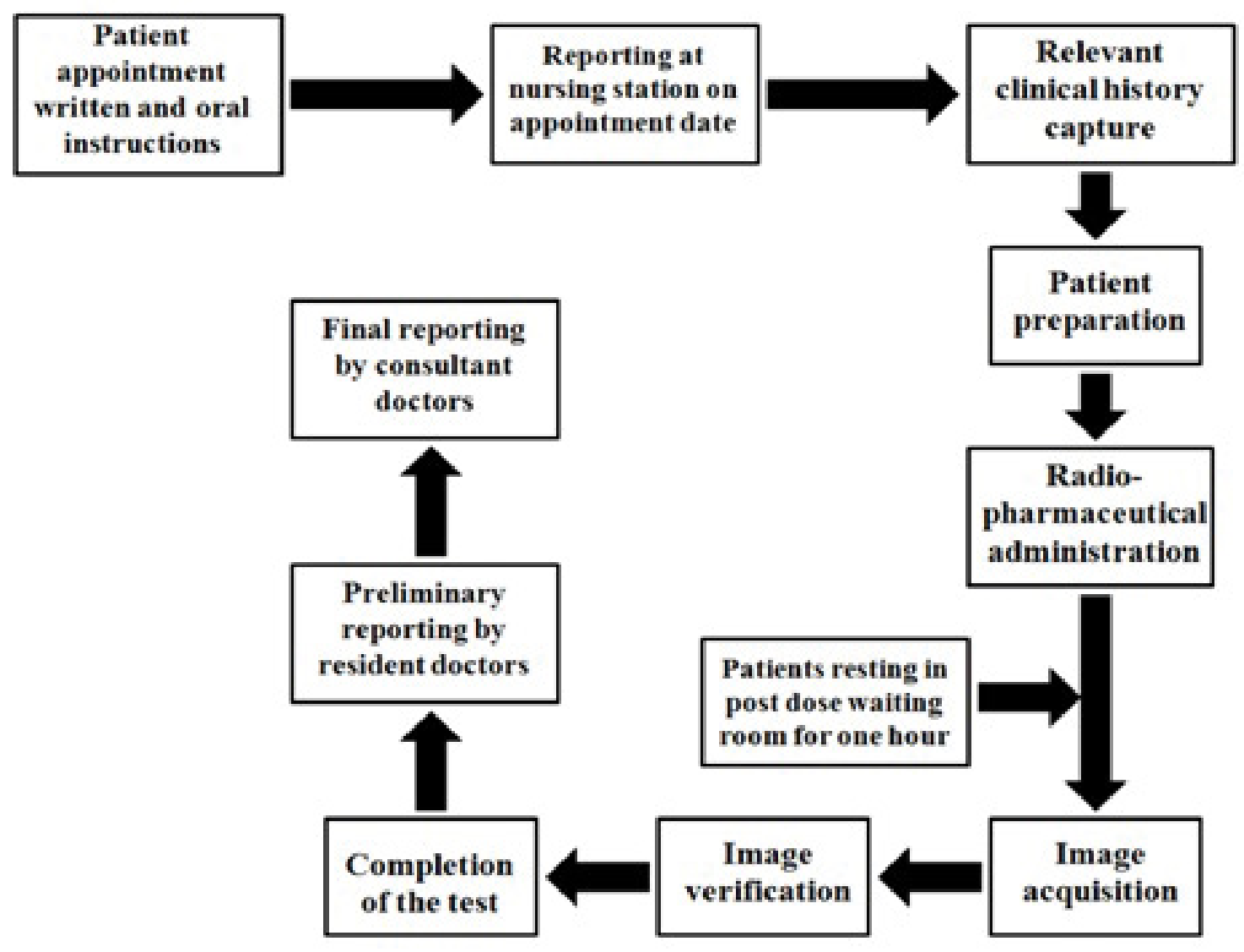

Figure 9: Workflow inside the PET/CT Facility. 
Descriptive analysis was applied to describe the characteristics of respondents in this study by percentage and frequency.

\section{Results}

A cross-sectional survey was conducted to 88 patients who were scheduled for a PET/ CT examination at Baghdad center. This survey showed that a $40.9 \%(36 / 88)$ of the participants was significantly concerned about the lack of dedicated clear protocol of the PET/CT examination. However, few of the participants understood what the assessment was or how it was conducted. Most the participants were significantly dissatisfied about being on the waiting list for prolonged time periods. $87.5 \%(77 / 88)$ of the participants wanted to have inpatient PET/CT scan done within less than one month. From which $64.8 \%$ (57/88) would like to have their examination within the 24 hours of request, while $6.8 \%(6 / 88)$ and $28.4 \%(25 / 88)$ of the participants wanted to have their scans within two weeks and one week of their request respectively. Though a portion of the participants didn't feel that the staff conveyed in a justifiable manner, $79.6 \%(70 / 88)$ of the participants was satisfied with the consideration provided by the center's staff and its professional skills, All the participants supported the idea that new PET/CT facility at Baghdad Medical City will significantly impact the management of patient and reduce both cost and potential associated with their travelling to the facilities existed in the neighboring countries. Such findings might be utilized to help optimise such medical protocols and patient consideration as well.

\section{Discussion}

$\mathrm{PET} / \mathrm{CT}$ is assuming an expanding role in cancer management. Similar to traditional Nuclear Medicine, Positron Emission Tomography (PET) studies are typically performed using higher-level radioactive materials injected and then the energy from the radioactive materials is measured giving indications of concentration and distribution of metabolic function. Contemporary PET scanners are now exclusively dual-modality scanners, such as $\mathrm{PET} / \mathrm{CT}$ or PET/MRI, combining the function of PET imaging and either $\mathrm{CT}$ or MRI into a single scanner. Because PET makes use of higher-level radioactive materials, access controls and security are required for both occupant safety and radioactive material security [10].
The primary goal of this study was to review the planning and design features considered to provide adequate safety and satisfaction measures of patients undergoing PET/CT imaging in local health care facilities, in order to improve and maintain the quality of clinical data acquired using such critical medical modality. Patient satisfaction is often employed as a primary measure of quality in PET/ CT facilities, and it encompasses both clinical and physical factors. Patient satisfaction represents a powerful quality measure since it reflects the facility's performance feasibility. Therefore, understanding the patient experience is critical since it allows for improvement.

According to the IAEA guidelines, major specialized equipment, as $\mathrm{PET} / \mathrm{CT}$, requires special structural design requirements that should be considered. New PET/CT facilities can proficiently utilize cement to accomplish required protecting factors. All the walls of the PET/CT facility should be made of thick brick or thick concrete but the walls of the PET/CT room should be concrete only, the thickness of which depends on the area and workload. In existing facilities, lead is frequently the best option. Portable lead shields can be utilized viably to shield patients in uptake rooms where they are needed to remain stationary. Lead shields 2.5 and $5.0 \mathrm{~cm}$ thick are commercially available, providing dose reduction factors of 40 and 1900, respectively. Utilizing shields in the scan room might be more hazardous in light of the fact that the patient is moved by the gantry and along these lines moves concerning the shield. Furthermore the shields can limit admittance to the patient.

Anticipating new facilities ought to carefully consider the requirements related with as far as possible. Uncontrolled areas with high occupancy ought to be situated as a long way from the uptake and imaging rooms as could reasonably be expected. Additionally, the placement of the door must be carefully considered to avoid the cost with introducing a door with substantial lead shielding.

In the event that uncontrolled areas are situated above and below the PET uptake and scan rooms, the separating between floors ought to be more prominent than typical. In the event that that isn't practical, the floors should be capable support the weight related with extra protecting. Floors and ceilings regularly have $10 \mathrm{~cm}$ of concrete, which will give about two half value layers for 


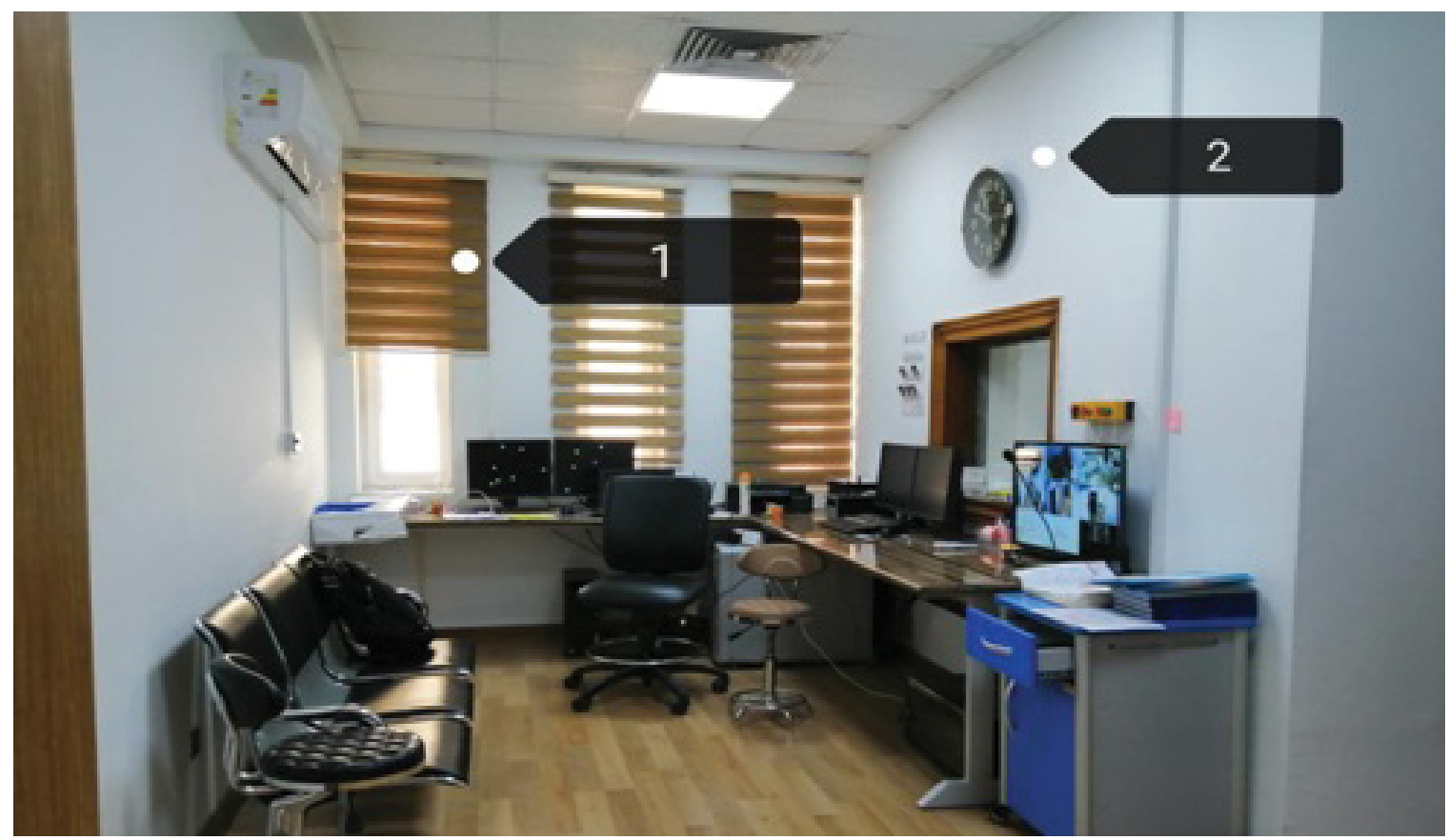

Figure 10: Walls were razed for the delivery of PET/CT: 1) The wall of control room to the outside; and 2) The wall between both control and scan rooms.

dose reduction. Walls will require 0.125 to 0.5 inch lead by and large. Doors will require 0.25 to 0.5 inches lead. For door with lead $>0.25$ inches, mechanical assist openers will be required. Utilize the lower occupancy factors for areas promptly outside doors to limit lead requirements for doors - attempt to keep doors to 0.25 inches lead or less. Viewing window must be leaded glass or acrylic to coordinate encompassing walls [11].

It should be understood that the default composition of a PET/CT clinical area should be in the form of a suite to facilitate the use of access control mechanisms required by radioactive materials licensure, accreditation requirements, and industry standards. Hot lab functions, as well as short term decay storage functions should be secured. When serving multiple nuclear medicine modalities, shared hot lab spaces should generally be located closer to the modalities that use the higher-energy radioisotopes reducing travel time and distance [10].

The challenge of introducing and starting clinical activities of PET/CT in a hospital requires cooperation from a multidisciplinary team. At the point when not just the PET/CT modality is new to the department, yet in addition the whole facility is new, much more prominent endeavors are needed so as to effectively start clinical action
[12]. In the PET/CT facility of Baghdad center, some challenges were indicated. The corridors were not wide enough when the device components were entered to the centre. So, the walls of the control room had been razed, one of them was overlooked to the outside and the other between both control and scan rooms. Then, by using the cranes they lifted it to the scan room, see Figure 10.

The scan room was not large enough and it should be expanded in order to achieve the operator's workflow in both sided of the gantry and as the control room does not required larger area so they expand the scan room at the expense of the control room, see Figure 11.

The other problem was the HVAC condition especially at summer as the average temperature rates increased and that may cause a problem in the working of the device especially for the motors, so in addition to the central cooling system they had to provide comfortable working environment for the staff and the patients in order to minimize any disturbing factors for the imaging procedure.

In Baghdad center, the PET/CT equipment was installed in the second floor. This indicates difficulty in the entry and exit of the isotopes. Also the centre does not provide the 24 hours overnight stay for the patients, even with lower doses. This may contribute to the environmental pollution. 


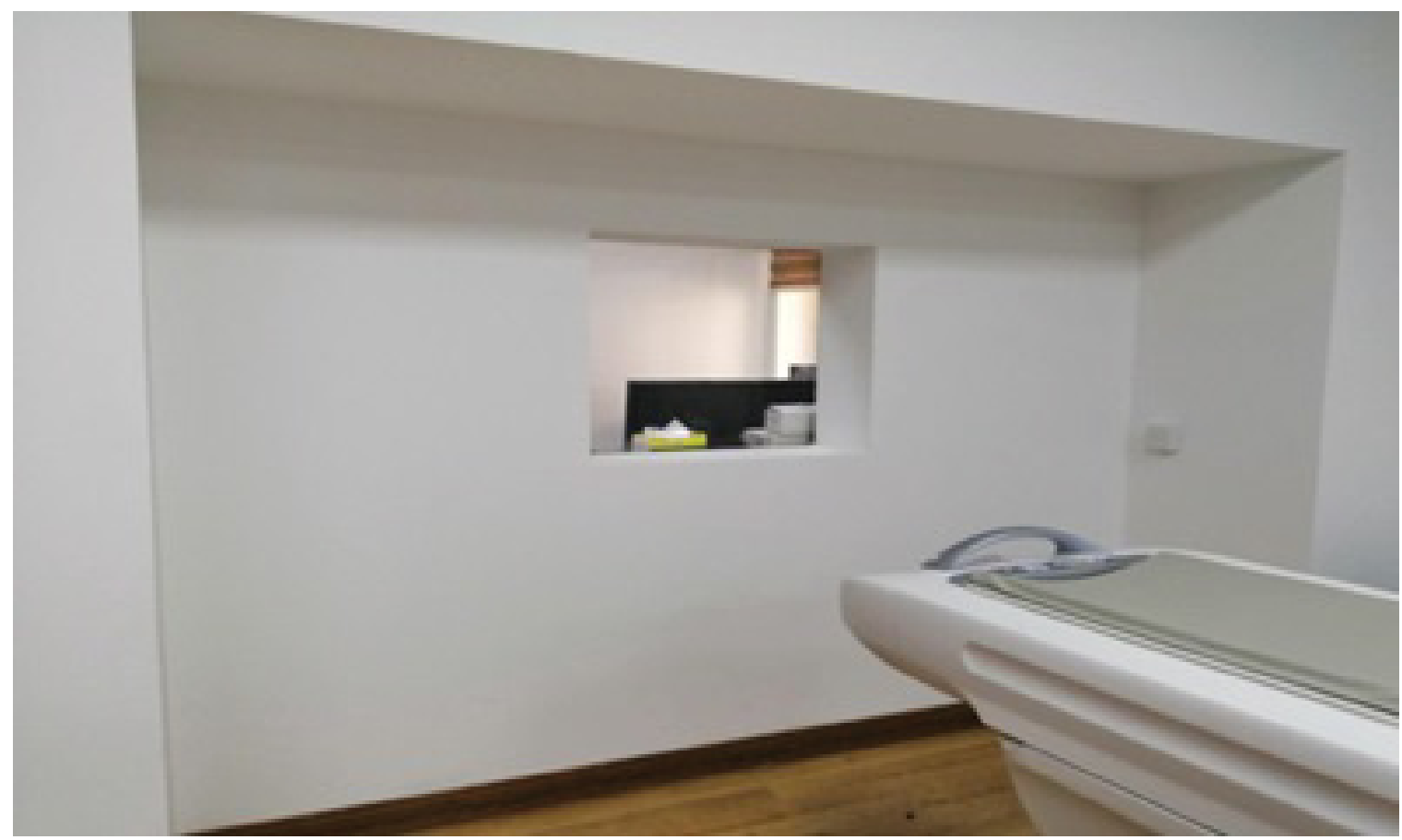

Figure 11: The expansion of the scan room.

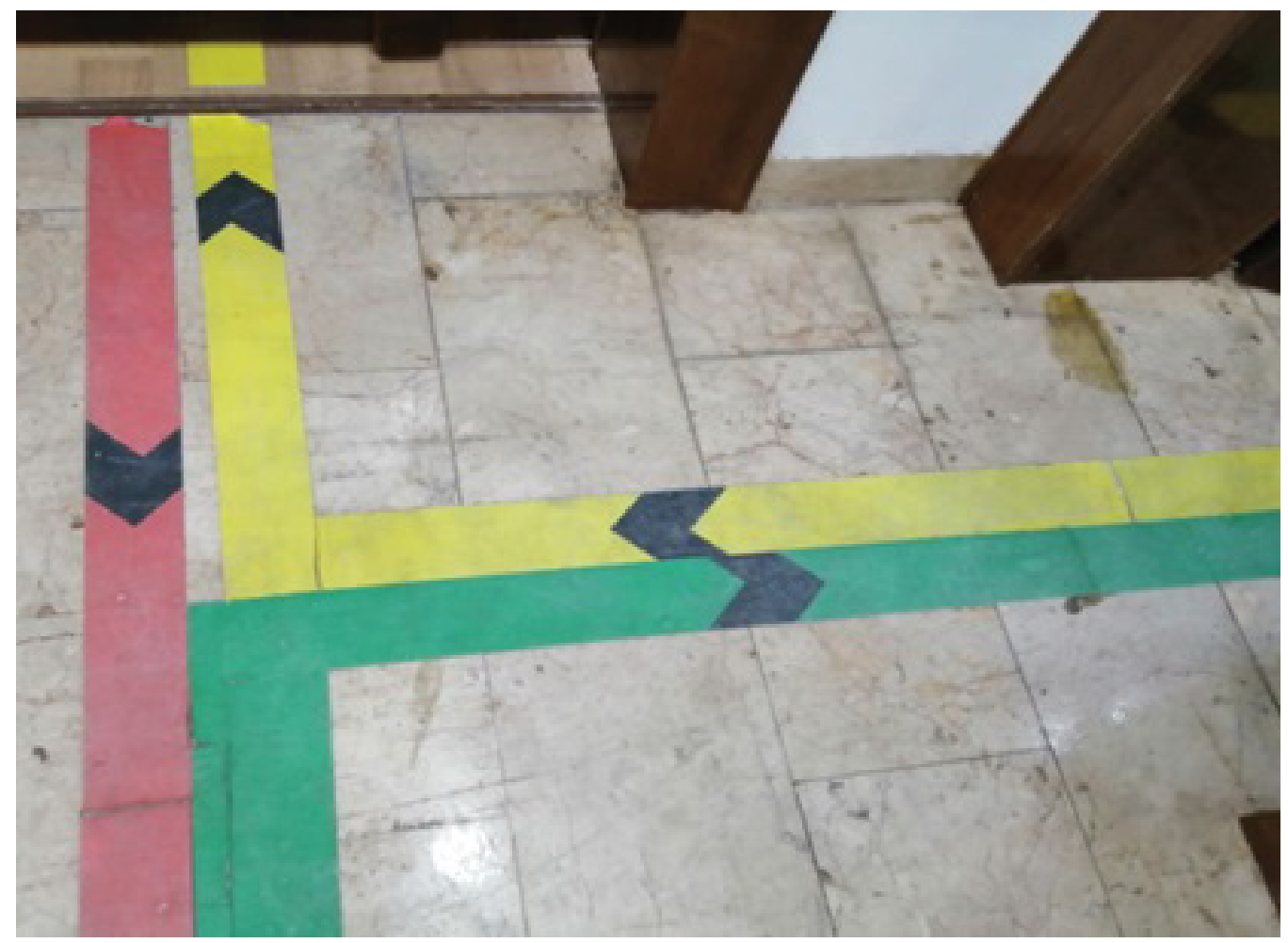

Figure 12: The colours used to identify patient destination.

The ground was lined with three colours in order to identify the patients' destination and guide them according to their condition. So, the red colour was employed for guiding patients after scanning, the green colour for guiding patients before injection and the yellow colour for guiding injected patients who are waiting for the scanning, see Figure 12. This step indicates many benefits as the patient will know where to go without mingle with the other people in the hospital. 
The PET/CT assessment requires arranging and readiness with respect to both patients and medical staff. Accordingly, it is significant that patient should be well informed and that the medical staff should be all around prepared. The review study indicate that the staff of Baghdad center applied a proper protection procedure against radioactive contamination by using proper clothing and dosimeters, maintaining distance from the radiation source and keeping the time of exposure to a minimum. Through providing proper handling facilities and following good work rehearses, radiation dose to the staff, public, and environment can be kept up well beneath as far as possible. It would be more beneficial if the facility is involved within a nuclear medicine department where the space could be saved as many rooms are common with larger staff number can be effective in order to achieve better clinical service.

This study was not without limitations; the influence of restrictions beyond the COVID-19 pandemic has had an impact on the low rate of survey participation, since the relevant medical institution has limited in- person activities throughout the period of conducting this research, limiting the enrolled sample size to only 88 individuals. Meanwhile, past research indicates that this is the first time patients' knowledge and attitudes have been assessed at PET/CT healthcare facilities in Iraq, which encourages such research investigation. This could serve as a useful foundation for future research in order to generate better facilities planning and design.

\section{Conclusion}

$\mathrm{PET} / \mathrm{CT}$ is playing an increasing role in cancer management. Its facilities present somewhat special requirements than conventional nuclear medicine facilities. It requires careful planning and designing to facilitate the traffic of patients, staff and radioactive materials and is more likely to require additional shielding. The obtained findings of this study show that more potential need to done in order to enhance the performance of the PET/CT facilities in Baghdad center. Quality control concepts must be done according to the recommended standards on regular basis in order to achieve an excellent clinical service besides the safety of both staff and patients. Constructing a cyclotron for the $\mathrm{PET} / \mathrm{CT}$ requires enormous efforts since there are so many constraints. The major constraint that needs to be settled is about the budget and its cost benefits. For some developing countries, it would be difficult to manage a policy including the construction of the PET/CT facility. The physicians, radiologists and technologists involved must be well educated and trained in PET/CT imaging procedures and radiation protection principles.

Moreover, guidelines fulfillment as far as possible for uncontrolled zones can be a costly recommendation. Proper planning of the equipment merchant, facility architect, and a qualified medical physicist is important to create a cost feasible design while keeping up radiation safety standards. These guidelines must be actualized through a successful radiation safety infrastructure that incorporates sufficient laws and guidelines, a proficient administrative framework, supporting specialists and administrations, and a 'safety culture' shared by every one of those with responsibilities for protection, including both management and workers. It is highly recommended to introduce new PET/CT facilities in the future to reduce the load on the recent exist unit in Baghdad city with considering that it must planned and designed on the basis of regulatory requirements and radiation safety standards. It has conclusively shown that further research is required to identify which information demands are crucial for improving the cancer care in Iraq.

\section{Acknowledgement}

The administrative and technical staff of Baghdad Center for Radiation Therapy and Nuclear Medicine of Baghdad Medical City.

\section{References}

1. Risdianto NS, Purwanto J, Rahmadi FA (2016) The use of cyclotron for PET/CT scan in Indonesian hospitals and future collaboration. Proceedings of IPAC, Busan, Korea.

2. International Atomic Energy Agency (2010) IAEA human health series: Planning a clinical PET centre. Series no. 11, Vienna, Austria.

3. Radiological Protection Institute of Ireland (2008) The design of diagnostic medical facilities using ionizing radiation. $\left(2^{\text {nd }} e d n\right)$, Dublin, Ireland.

4. International Atomic Energy Agency (1996) IAEA International basic safety standards for protection against lonising radiation and for the safety of radiation sources. Safety series no. 115, Vienna, Austria. 
5. Valentin J (2007) The 2007 recommendations of the international commission on radiological protection. ICRP publication 103. Ann ICRP 37: 1-332.

6. Malone J (2008) Radiation protection in newer medical imaging techniques: PET/CT. IAEA safety report series no. 58, Vienna, Austria.

7. International Health Facility Guidelines (2017) Part B- Healthy facility briefing and design. Version 5, Australia.

8. Bresnahan ME, Shrestha B (2012) Potential shielding for a positron emission tomography (PET) suite. Journal of Biomedical Graphics and Computing 2: 8995.
9. Jha AK, Singh AM, Mithun S, Shah S, Agrawal A, et al. (2015) Designing of high volume PET/CT facility with optimal reduction of radiation exposure to the staff: Implementation and optimization in a tertiary health care facility in India. World J Nucl Med 14: 189-196.

10.U.S. Department of Veterans Affairs (2020) PG 18-12 - Imaging services design guide. Chapter 295, USA.

11. Madsen MT, Anderson JA, Halama JR, Kleck J, Simpkin DJ, et al. (2006) AAPM task group 108: PET and PET/ CT shielding requirements. Med Phys 33: 4-15.

12. Hagland J (2016) Preparing for clinical drift of PET/ $\mathrm{CT}$, An example from Norway. RAD Conference Proceedings 1: 106-110. 